Pacific Journal of Mathematics

EXTENSIONS OF VALUATION AND ABSOLUTE VALUED 


\title{
EXTENSIONS OF VALUATION AND ABSOLUTE VALUED TOPOLOGIES
}

\author{
JO-ANN D. COHEN
}

It is known that if $L$ is a separable, finite dimensional extension of a field $K$ and if $v$ is a proper valuation (absolute value) on $K$, then each ring topology on $L$ whose restriction to $K$ is the topology $\mathscr{T}_{v}$ defined on $K$ by $v$ is the supremum of a finite family of valuation (absolute valued) topologies. We give a characterization of the fields $K$ and $L$ and the valuations (absolute values) $v$ on $K$ for which each ring topology on $L$ extending $\mathscr{T}_{v}$ is the supremum of a family of valuation (absolute valued) topologies on $K$ when $L$ is an arbitrary finite dimensional extension of $K$.

Let $R$ be a ring and let $\mathscr{T}$ be a ring topology on $R$, that is, $\mathscr{T}$ is a topology on $R$ making $(x, y) \rightarrow x-y$ and $(x, y) \rightarrow x y$ continuous from $R \times R$ to $R$. A subset $A$ of $R$ is bounded for $\mathscr{T}$ if given any neighborhood $U$ of zero, there exists a neighborhood $V$ of zero such that $V A \cup A V$ $\subseteq U . \mathscr{T}$ is a locally bounded topology on $R$ if there exists a fundamental system of neighborhoods of zero for $\mathscr{T}$ consisting of bounded sets.

Recall that a norm $N$ on a ring $R$ is a function from $R$ to the nonnegative reals satisfying $N(x)=0$ if and only if $x=0, N(x-y) \leq$ $N(x)+N(y)$ and $N(x y) \leq N(x) N(y)$ for all $x$ and $y$ in $R$. Each norm $N$ on $R$ defines a locally bounded topology $\mathscr{T}_{N}$ on $R$ in a natural way. In particular, if $|\cdot|$ is a proper absolute value on a field $K$, then there exists a locally bounded topology $\mathscr{T}_{|. .|}$on $K$ defined by $|\cdot| \cdot$. We note further that if $N$ is a nontrivial norm on a field $K$, that is, $\mathscr{T}_{N}$ is nondiscrete, then a subset $A$ of $K$ is bounded in norm if and only if $A$ is a $\mathscr{T}_{N}$-bounded subset of $K$.

Each proper valuation $v$ on a field $K$ defines a locally bounded topology $\mathscr{T}_{v}$ on $K$ as well. If each of $v$ and $w$ is a valuation or an absolute value on $K$, then $v$ and $w$ are independent if $\mathscr{T}_{v} \neq \mathscr{T}_{w}$.

In [11], Rigo and Warner proved that if $L$ is a separable, finite dimensional extension of a field $K$ and if $v$ is a proper valuation (absolute value) on $K$, then each ring topology on $L$ inducing $\mathscr{T}_{v}$ on $K$ is the supremum of a finite family of valuation (absolute valued) topologies on $L$ (Theorem 2). In this paper we characterize the fields $K$ and $L$ and valuations (absolute values) $v$ on $K$ for which each ring topology on $L$ 
extending $\mathscr{T}$, is the supremum of a finite family of valuation (absolute valued) topologies on $L$ when $L$ is an arbitrary finite dimensional extension of $K$.

THEOREM 1. Let $K$ be a field, $v$ a proper valuation (absolute value) on $K, \hat{K}$ the completion of $K$ for $\mathscr{T}_{v}, L$ a purely inseparable, finite dimensional extension of $K, w$ the unique extension of $v$ to $L$ and $\hat{L}$ the completion of $L$ for $\mathscr{T}_{w}$. The following are equivalent.

$1^{\circ} \cdot[\hat{L}: \hat{K}]=[L: K]$.

$2^{\circ} . \mathscr{T}_{w}$ is the only ring topology on $L$ whose restriction to $K$ is $\mathscr{T}_{v}$.

$3^{\circ} . \mathscr{T}_{w}$ is the only locally bounded topology on $L$ whose restriction to $K$ is $\mathscr{T}_{v}$.

Proof. We first consider the case when $[L: K]=p$ where $p$ is the characteristic of $K$.

Suppose $[\hat{L}: \hat{K}]=p$. Then there exists a in $L \backslash \hat{K}$. Hence $L=K(a)$ and the minimal polynomial of a over $K$ is irreducible in $\hat{K}[X]$. Thus by [11, Corollary 2 of Theorem 1], $\mathscr{T}_{w}$ is the only ring topology on $L$ whose restriction to $K$ is $\mathscr{T}_{v}$.

Clearly $2^{\circ}$ implies $3^{\circ}$. So it suffices to prove that if $\mathscr{T}_{w}$ is the only locally bounded topology on $L$ whose restriction to $K$ is $\mathscr{T}_{v}$, then $[\hat{L}: \hat{K}]=p$. If $[\hat{L}: \hat{K}]=1$, let $a \in L \backslash K$ and let $f(X)$ be the minimal polynomial of a over $K$. Then $f(X)=(X-a)^{p}$ and $X-a \in \hat{K}[X]$. Hence by [11, Theorem 1], there are $p$ ring topologies $\mathscr{T}_{1}, \ldots, \mathscr{T}_{p}$ on $L$ inducing $\mathscr{T}_{v}$ on $K$ and the completion $\hat{L}_{i}$ of $L$ for $\mathscr{T}_{l}$ is a finite dimensional $\hat{K}$-algebra for each $i \in[1, p]$. If $v$ is a valuation on $K$, let $\hat{v}$ be its extension to $\hat{K}$, let $G$ be the order group of $\hat{v}$ and let $\left\{x_{1}, \ldots, x_{n}\right\}$ be a basis for $\hat{L}_{i}$ over $\hat{K}$ where $x_{1}=1$. Then $\left\{V_{\alpha}: \alpha \in G\right\}$ is a fundamental system of neighborhoods of zero for a Hausdorff topology on $\hat{L}_{l}$ compatible with the vector space structure of $\hat{L}_{i}$ where for each $\alpha \in G$,

$$
V_{\alpha}=\left\{\sum_{j=1}^{n} a_{j} x_{J}: a_{J} \in \hat{K}, \inf \left\{\hat{v}\left(a_{j}\right): 1 \leq j \leq n\right\} \geq \alpha\right\} .
$$

Hence by [8, Theorem 7], $\left\{V_{\alpha}: \alpha \in G\right\}$ is a fundamental system of neighborhoods of zero for the completion $\hat{\mathscr{T}}_{i}$ of $L$ for $\mathscr{T}_{i}$. It follows that the restriction of $\hat{\mathscr{T}}_{i}$ to $\hat{K}$ is the topology defined on $\hat{K}$ by $\hat{v}$. Thus as $L \subseteq \hat{K},\left.\hat{\mathscr{T}}_{i}\right|_{L}$ is a locally bounded topology on $L$, that is, each $\mathscr{T}_{l}$ is a locally bounded topology on $L$, a contradiction. If $v$ is an absolute value on $K$, then each $\mathscr{T}_{1}$ is normable and hence locally bounded. Indeed, by [2, Theorem 2, p. 27; 3, Proposition 10, p. 69 and Theorem 1, p. 70], there exist a vector space norm $N$ on $\hat{L}_{i}$ and a positive number $c$ such that 
$N(x y) \leq c N(x) N(y)$ for all $x$ and $y$ in $\hat{L}_{l}$. Therefore the function $\|\cdot\|$ defined on $\hat{L}_{i}$ by, $\|x\|=c N(x)$, is an algebra norm on $\hat{L}_{i}$ defining the topology on $\hat{L}_{i}$. So $[\hat{L}: \hat{K}]=p$ by $[11$, Corollary 1 of Theorem 1$]$.

Now let $L$ be an arbitrary, purely inseparable, finite dimensional extension of $K$.

Suppose that $[\hat{L}: \hat{K}]=[L: K]$ and let $\mathscr{T}$ be a ring topology on $L$ whose restriction to $K$ is $\mathscr{T}_{v}$. Let $K_{1}$ be a maximal subfield of $L$ containing $K$ such that $\left.\mathscr{T}\right|_{K_{1}}$ is defined by a valuation (absolute value) $v_{1}$ extending $v$ to $K_{1}$. If $K_{1} \neq L$, let $a \in L \backslash K_{1}$ be such that $\left[K_{1}(a): K_{1}\right]=$ $p$. Denote $K_{1}(a)$ by $K_{2}$, let $v_{2}$ be the unique extension of $v$ to $K_{2}$, let $\hat{K}_{1}$ be the completion of $K_{1}$ for $\mathscr{T}_{v_{1}}$ and let $\hat{K}_{2}$ be the completion of $K_{2}$ for $\mathscr{T}_{v_{2}}$. If $a \notin \hat{K}_{1}$, then by the previous argument, $\left.\mathscr{T}\right|_{K_{2}}=\mathscr{T}_{v_{2}}$, contradicting the maximality of $K_{1}$. If $a \in \hat{K}_{1}$, then $\left[\hat{K}_{2}: \hat{K}_{1}\right]=1$. So $[\hat{L}: \hat{K}]=$ $\left[\hat{L}: \hat{K}_{2}\right]\left[\hat{K}_{1}: \hat{K}\right] \leq\left[L: K_{2}\right]\left[K_{1}: K\right]<[L: K]$, a contradiction. Hence $K_{1}$ $=L$.

Assume $3^{\circ}$ holds. Let $[L: K]=p^{n}$ and let $a_{1}, a_{2}, \ldots, a_{n} \in L$ be such that $L=K\left(a_{1}, \ldots, a_{n}\right),\left[K\left(a_{1}\right): K\right]=p$ and for all $i \in[1, n-1]$, $\left[K\left(a_{1}, \ldots, a_{i+1}\right): K\left(a_{1}, \ldots, a_{i}\right)\right]=p$. Denote $K$ by $K_{0}$. For each $i \in[1, n]$ let $K_{t}=K\left(a_{1}, \ldots, a_{i}\right)$, let $v_{i}$ be the unique extension of $v$ to $K_{i}$ and let $\hat{K}_{t}$ be the completion of $K_{i}$ for $\mathscr{T}_{v_{i}}$. If $p^{n}>[\hat{L}: \hat{K}]$, then as $[\hat{L}: \hat{K}]=$ $\prod_{i=0}^{n-1}\left[\hat{K}_{i+1}: \hat{K}_{i}\right]$, there exists an $i$ such that $\left[\hat{K}_{i+1}: \hat{K}_{i}\right]=1$. So by the previous argument there exists a locally bounded topology $\mathscr{T}^{\prime}$ on $K_{i+1}$ whose restriction to $K_{i}$ is $\mathscr{T}_{v_{i}}$ but $\mathscr{T}^{\prime} \neq \mathscr{T}_{v_{t+1}}$. By [12, Satz 1.6], $\mathscr{T}^{\prime}$ extends to a locally bounded topology $\mathscr{T}$ on $\stackrel{L}{ }$. Clearly $\left.\mathscr{T}\right|_{K}=\mathscr{T}_{v}$ but $\mathscr{T} \neq \mathscr{T}_{w}$, a contradiction. So $[\hat{L}: \hat{K}]=p^{n}=[L: K]$.

TheOREM 2. Let $L$ be a finite dimensional extension of a field $K$, let $D$ be the separable closure of $K$ in $L$, let $v$ be a proper valuation (absolute value) on $K$ and let $\left\{v_{i}: 1 \leq i \leq m\right\}$ be a complete family of pairwise independent valuations (absolute values) on $D$ extending $v$. For each $i \in[1, m]$, let $w_{i}$ be the unique extension of $v_{i}$ to $L$, let $\hat{L}_{i}$ denote the completion of $L$ for $\mathscr{T}_{w_{i}}$ and let $\hat{D}_{i}$ denote the completion of $D$ for $\mathscr{T}_{v_{i}}$. The following are equivalent.

$1^{\circ}$. Each ring topology on $L$ whose restriction to $K$ is $\mathscr{T}_{v}$ is the supremum of a finite family of valuation (absolute valued) topologies on $L$.

$2^{\circ}$. Each locally bounded topology on $L$ whose restriction to $K$ is $\mathscr{T}_{v}$ is the supremum of a finite family of valuation (absolute valued) topologies on $L$.

$3^{\circ}$. There are $2^{m}-1$ locally bounded topologies on $L$ inducing $\mathscr{T}_{v}$ on $K$, namely the topologies $\sup _{i \in M} \mathscr{T}_{w_{t}}$ where $M$ runs through all nonempty subsets of $[1, m]$. 


$$
4^{\circ} \cdot\left[\hat{L}_{i}: \hat{D}_{i}\right]=[L: D] \text { for all } i \in[1, m] \text {. }
$$

Proof. Clearly $1^{\circ}$ implies $2^{\circ}$ and $3^{\circ}$ implies $2^{\circ}$. We first show that $2^{\circ}$ implies $3^{\circ}$. Suppose that $\mathscr{T}$ is a locally bounded topology on $L$ and $\mathscr{T}=\sup _{1 \leq i \leq n} \mathscr{T}_{u_{i}}$ where each $u_{i}$ is a proper valuation (absolute value) on $L$ and $\mathscr{T}_{u_{i}} \neq \mathscr{T}_{u_{j}}$ for $i \neq j$. Then $\mathscr{T}_{v}=\mathscr{T}_{\left.\right|_{K}}=\left.\sup _{1 \leq i \leq n} \mathscr{T}_{u_{i}}\right|_{K}$. As the completion of $K$ for $\mathscr{T}_{v}$ is a field, the Approximation Theorem [7, Theorem 3.4, p. 292] yields that each $\left.u_{i}\right|_{K}$ is equivalent to $v$. Hence for each $i \in[1, n]$, there exists $j(i) \in[1, m]$ such that $\mathscr{T}_{u_{t}}=\mathscr{T}_{w_{j(t)}}$.

We next show that $3^{\circ}$ implies $4^{\circ}$. Let $\mathscr{T}$ be a locally bounded topology on $L$ whose restriction to $D$ is $\mathscr{T}_{v_{t}}$ and let $M$ be a nonempty subset of $[1, m]$ such that $\mathscr{T}=\sup _{j \in M} \mathscr{T}_{w}$. Note that for $\iota, j \in M, \iota \neq j$, $\left.\mathscr{T}_{w_{\imath}}\right|_{D} \neq\left.\mathscr{T}_{w_{j}}\right|_{D}$. Then $\mathscr{T}_{v_{i}}=\left.\mathscr{T}\right|_{D}=\left.\sup _{j \in M} \mathscr{T}_{w_{j}}\right|_{D}$ and so the Approximation Theorem implies that the cardinality of $M$ is one. Thus $M=\{i\}$ by the definition of $w_{i}$, that is, $\mathscr{T}=\mathscr{T}_{w_{i}}$. As $L$ is a purely inseparable extension of $D, 4^{\circ}$ follows from Theorem 1 .

Finally suppose that $4^{\circ}$ holds. Let $\mathscr{T}$ be a ring topology on $L$ whose restriction to $K$ is $\mathscr{T}_{v}$. By Theorems 2 and 4 of [11], there exist a nonempty subset $M$ of $[1, m]$ and ring topologies $\mathscr{T}_{i}$ on $L$ for each $i \in M$ such that $\left.\mathscr{T}_{i}\right|_{D}=\mathscr{T}_{v_{i}}$ and $\mathscr{T}=\sup _{i \in M} \mathscr{T}_{i}$. Hence $\mathscr{T}_{i}=\mathscr{T}_{w_{i}}$ for all $i \in M$ by Theorem 1 and so $1^{\circ}$ holds.

COROLLARY. Let $K$ be the field $F(X)$ of rational functions over the field $F$, let $L$ be a finite dimensional extension of $K$ and let $v$ be a proper valuation or absolute value on $K$, improper on $F$. Define $D, L_{i}$ and $\hat{D}_{i}$ as in Theorem 2. Then $\left[\hat{L}_{i}: \hat{D}_{i}\right]=[L: D]$ for all $i$ and each ring topology on $L$ inducing $\mathscr{T}_{v}$ on $K$ is a locally bounded topology.

Proof. First note that if $v$ is a proper valuation on $K$ improper on $F$, then $v$ is equivalent to a real-valued valuation [1, Example 4, p. 106]. It suffices to establish $2^{\circ}$ of Theorem 2 . Let $\mathscr{T}$ be a locally bounded topology on $L$ whose restriction to $K$ is $\mathscr{T}_{v}$. Then there exists a nonzero topological nilpotent for $\mathscr{T}_{v}$ and hence for $\mathscr{T}$. So by [5, Theorem 6.1], there exists a norm $N$ on $L$ such that $\mathscr{T}=\mathscr{T}_{N}$. As $F$ is a bounded subset of $K$ for $\mathscr{T}_{v}$ and as $\left.\mathscr{T}_{N}\right|_{K}=\mathscr{T}_{v}, F$ is bounded in norm (for $N$ ). Consequently, $F$ is a $\mathscr{T}$-bounded subset of $L$ as well. Thus by [6, Theorem 4] and the argument used to establish Theorem 3 of [4], $\mathscr{T}$ is the supremum of a finite family of valuation topologies on $L$.

In [9], Nagata gave an example of fields $L$ and $K$, each of prime characteristic $p$, and a discrete valuation $v$ on $K$ such that $\hat{K}=L$ is a 
purely inseparable extension of $K$ of degree $p$ over $K$ (p. 56). Thus $\hat{L}=\hat{K}$ and so conditions $1^{\circ}-4^{\circ}$ of Theorem 2 need not hold in general.

TheOREM 3. Let $K$ be a field and let $\mathscr{T}_{0}$ denote $\sup _{1 \leq i \leq m} \mathscr{T}_{v_{1}}$ where each $v_{i}$ is a proper valuation or absolute value on $K$ and for $i \neq j, \mathscr{T}_{v_{t}} \neq \mathscr{T}_{v_{j}}$. Let $L$ be a finite dimensional extension of $K$ and let $D$ be the separable closure of $K$ in $L$. For each $i \in[1, m]$, let $\left\{v_{i j}: 1 \leq j \leq M(i)\right\}$ be a complete family of pairwise independent valuations or absolute values extending $v_{i}$ to $D$. For each $i \in[1, m], j \in[1, M(i)]$, let $w_{i j}$ denote the unique extension of $v_{i j}$ to $L$, let $\hat{L}_{i j}$ denote the completion of $L$ for $\mathscr{T}_{w_{i j}}$ and let $\hat{D}_{i j}$ denote the completion of $D$ for $\mathscr{T}_{v_{i}}$. The following are equivalent.

$1^{\circ}$. Each ring topology on $L$ whose restriction to $K$ is $\mathscr{T}_{0}$ is the supremum of a finite family $\left\{\mathscr{T}_{1}, \ldots, \mathscr{T}_{n}\right\}$ of topologies on $L$ where for each $i, \mathscr{T}_{i}$ is defined by a proper valuation or absolute value on $L$.

$2^{\circ}$. Each locally bounded topology on $L$ whose restriction to $K$ is $\mathscr{T}_{0}$ is the supremum of a finite family $\left\{\mathscr{T}_{1}, \ldots, \mathscr{T}_{n}\right\}$ of topologies on $L$ where for each $i, \mathscr{T}_{i}$ is defined by a proper valuation or absolute value on $L$.

$3^{\circ}$. There are $\Pi_{i=1}^{m}\left(2^{M(i)}-1\right)$ locally bounded topologies on $L$ inducing $\mathscr{T}_{0}$ on $K$, namely the topologies $\sup _{1 \leq i \leq m}\left(\sup _{j \in S(i)} \mathscr{T}_{w_{i j}}\right)$ where $S(i)$ runs through all nonempty subsets of $[1, M(i)]$.

$4^{\circ} .\left[\hat{L}_{i j}: \hat{D}_{i j}\right]=[L: D]$ for all $i \in[1, m], j \in[1, M(i)]$.

Proof. Clearly $1^{\circ}$ implies $2^{\circ}$ and $3^{\circ}$ implies $2^{\circ}$. We first prove that $2^{\circ}$ implies $3^{\circ}$. Let $\mathscr{T}$ be a locally bounded topology on $L$ inducing $\mathscr{T}_{0}$ on $K$. Then $\mathscr{T}=\sup _{1 \leq i \leq n} \mathscr{T}_{u_{t}}$ where each $u_{i}$ is a proper valuation or absolute value on $L$ and $\sup _{1 \leq j \leq m} \mathscr{T}_{v_{1}}=\left.\sup _{1 \leq \iota \leq n} \mathscr{T}_{u_{t}}\right|_{K}$. Suppose that there exists an $i, 1 \leq i \leq n$, such that for all $j, 1 \leq j \leq m,\left.\mathscr{T}_{u_{i}}\right|_{K} \neq \mathscr{T}_{v}$. Without loss of generality assume that $v_{1}, \ldots, v_{r}$ are valuations on $K$, $v_{r+1}, \ldots, v_{m}$ are absolute values on $K$ and $i=1$. If $u_{1}$ is an absolute value on $L$, let $a \in K$ be such that $u_{1}(a)>1, v_{j}(a)>0$ for $j \in[1, r]$ and $v_{j}(a)<1$ for $j \in[r+1, m]$. (The existence of $a$ is guaranteed by [7, Theorem 3.4, p. 292].) Then $\left\{a^{t}: t=1,2, \ldots\right\}$ is a bounded set for $\sup _{1 \leq \jmath \leq m} \mathscr{T}_{v_{j}}$ but not for $\left.\mathscr{T}_{u_{1}}\right|_{K}$, a contradiction. (Indeed, if $\left\{a^{t}: t=\right.$ $1,2, \ldots\}$ is bounded for $\left.\sup _{1 \leq \imath \leq n} \mathscr{T}_{u_{l}}\right|_{K}$, then there exists a nonzero element $x$ in $K$ such that $x\left\{a^{t}: t=1,2, \ldots\right\} \subseteq\left\{y \in K: u_{1}(y) \leq 1\right\}$. But $u_{1}\left(x z^{t}\right) \rightarrow \infty$ as $t \rightarrow \infty$, a contradiction.) If $u_{1}$ is a valuation on $L$, let $G$ be the order group of $\left.u_{1}\right|_{K}$ and for each $\alpha \in G$, let $a_{\alpha} \in K$ be such that $v_{j}\left(a_{\alpha}\right)>0$ for $j=1,2, \ldots, r, v_{j}\left(a_{\alpha}\right)<1$ for $j=r+1, \ldots, m$ and $u_{1}\left(a_{\alpha}\right)$ $=\alpha$. Then $\left\{a_{\alpha}: \alpha \in G\right\}$ is a bounded set for $\sup _{1 \leq j \leq m} \mathscr{T}_{v_{j}}$ but not for $\left.\mathscr{T}_{u_{1}}\right|_{K}$, a contradiction. Thus for each $i \in[1, n]$ there exists $j(i) \in[1, m]$ 
and $t(i) \in[1, M(j(i))]$ such that $\mathscr{T}_{u_{t}}=\mathscr{T}_{w_{f(i), t(t)}}$. Furthermore a similar argument establishes that for each $j \in[1, m]$, there exists an $i \in[1, n]$ such that $\left.\mathscr{T}_{u_{i}}\right|_{K}=\mathscr{T}_{v}$.

Assume $3^{\circ}$ holds. Suppose that there exist $i \in[1, m]$ and $j \in[1, M(i)]$ with $\left[\hat{L}_{i j}: \hat{D}_{i j}\right]<[L: D]$. By Theorem 2 there exists a locally bounded topology $\mathscr{T}$ on $L$ whose restriction to $K$ is $\mathscr{T}_{v_{t}}$ but $\mathscr{T}$ is not the supremum of a finite family of valuation or absolute valued topologies on $L$. Let $\mathscr{T}^{\prime}=\sup \left(\mathscr{T}, \sup _{\iota \neq i} \mathscr{T}_{w_{t l}}\right)$. Then $\left.\mathscr{T}^{\prime}\right|_{K}=\mathscr{T}_{0}$ but $\mathscr{T}^{\prime}$ is not the supremum of a finite family of topologies on $L$ of the appropriate type. Indeed, if $\mathscr{T}^{\prime}$ is such a supremum, then as $\mathscr{T} \subseteq \mathscr{T}^{\prime}$, Theorem 4.4 of [10] yields that $\mathscr{T}$ is as well. Thus $4^{\circ}$ holds.

Finally the proof that $4^{\circ}$ implies $1^{\circ}$ is the same as that used in Theorem 2.

\section{REFERENCES}

[1] N. Bourbaki, Algèbre Commutative, Ch. 5-6, Hermann, Paris, 1964.

[2] __ Espaces Vectoriels Topologiques, Ch. 1, Hermann, Paris, 1953.

[3] _ Topologie Générale, Ch. 9, Hermann, Paris, 1958.

[4] J. Cohen, The strong approximation theorem and locally bounded topoplogies on $F(X)$, Pacific J. Math., 87 (1980), 59-63.

[5] P. M. Cohn, An invariant characterization of pseudo-valuations on a field, Proc. Cambridge Phil. Soc., 50 (1954), 159-177.

[6] M. Endo, The strong approximation theorem and locally bounded topologies of algebraic function fields, Comment. Math. Univ. St. Paul, 30 (1981), 77-86.

[7] W. A. J. Luxemburg, (editor), Applications of Model Theory to Algebra, Analysis, and Probability, Holt, Rinehart and Winston, New York, 1969.

[8] L. Nachbin, On strictly minimal topological division rings, Bull. Amer. Math. Soc., 55 (1949), 1128-1136.

[9] M. Nagata, On the theory of Henselian rings, Nagoya Math. J., 5 (1953), 45-57.

[10] A. Prestel and M. Ziegler, Model theoretic methods in the theory of topological fields, J. Riene Angew. Math., 299/300 (1978), 318-341.

[11] T. Rigo and S. Warner, Topologies extending valuations, Canad. J. Math., 30 (1978), 164-169.

[12] H. Weber, Topologische Charakterisierung globaler Körper und algebraischer Funktionerkörper in einer Variablen, Math. Ziet., 169 (1979), 167-177.

Received May 17, 1985. Written while the author was in residence at the University of Maryland. 


\section{PACIFIC JOURNAL OF MATHEMATICS EDITORS}

\author{
V. S. VARADARAJAN \\ (Managing Editor) \\ University of California \\ Los Angeles, CA 90024 \\ HERBERT ClEMENS \\ University of Utah \\ Salt Lake City, UT 84112 \\ R. FINN \\ Stanford University \\ Stanford, CA 94305
}

\author{
HERMANN FLASCHKA \\ University of Arizona \\ Tucson, AZ 85721 \\ RAMESH A. GANGOLLI \\ University of Washington \\ Seattle, WA 98195 \\ VAUghan F. R. JONES \\ University of California \\ Berkeley, CA 94720 \\ ROBION KIRBY \\ University of California \\ Berkeley, CA 94720
}

C. C. MOORE

University of California Berkeley, CA 94720

H. SAMELSON

Stanford University Stanford, CA 94305

HAROLD STARK

University of California, San Diego La Jolla, CA 92093

\section{ASSOCIATE EDITORS}
R. ARENS
E. F. BECKENBACH
B. H. NEUMANN
F. WOLF
K. YOSHIDA (1906-1982)

\section{SUPPORTING INSTITUTIONS}

UNIVERSITY OF ARIZONA

UNIVERSITY OF BRITISH COLUMBIA

CALIFORNIA INSTITUTE OF TECHNOLOGY

UNIVERSITY OF CALIFORNIA

MONTANA STATE UNIVERSITY

UNIVERSITY OF NEVADA, RENO

NEW MEXICO STATE UNIVERSITY

OREGON STATE UNIVERSITY
UNIVERSITY OF OREGON UNIVERSITY OF SOUTHERN CALIFORNIA

STANFORD UNIVERSITY

UNIVERSITY OF HAWAII

UNIVERSITY OF TOKYO

UNIVERSITY OF UTAH

WASHINGTON STATE UNIVERSITY

UNIVERSITY OF WASHINGTON 


\section{Pacific Journal of Mathematics}

Vol. 125, No. 1 September, 1986

Gilles Christol, Fonctions et éléments algébriques $\ldots \ldots \ldots \ldots \ldots \ldots \ldots \ldots$

Jo-Ann Deborah Cohen, Extensions of valuation and absolute valued

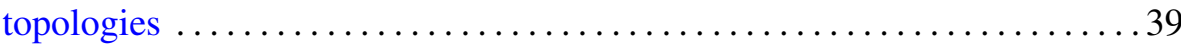

Miriam Cohen, Smash products, inner actions and quotient rings . . . . . . 45

Mikio Furushima, On the singular $K-3$ surfaces with hypersurface

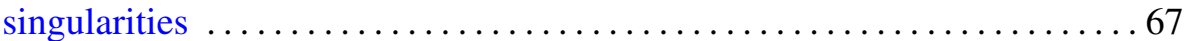

Gerhard Gierz and Boris Shekhtman, A duality principle for rational

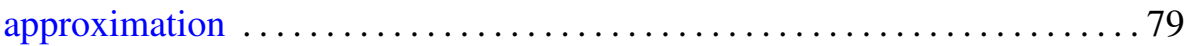

Anthony Wood Hager, A description of HSP-like classes, and

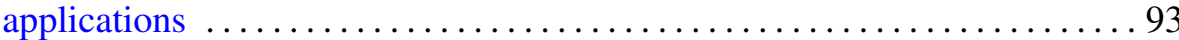

George Alan Jennings, Lines having high contact with a projective

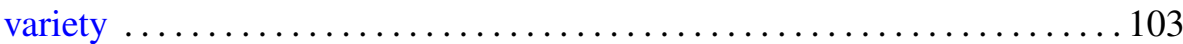

John Lott, Eigenvalue bounds for the Dirac operator . . . . . . . . . . . 117

Denis Laurent Luminet, A functional calculus for Banach PI-algebras . . . . 127

Shizuo Miyajima and Noboru Okazawa, Generators of positive

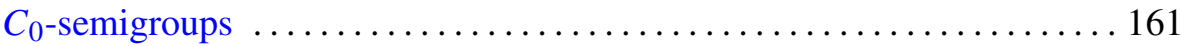

Takemi Mizokami, On functions and stratifiable $\mu$-spaces $\ldots \ldots \ldots \ldots \ldots 177$

Jeff Parker, 4-dimensional $G$-manifolds with 3-dimensional orbits . . . . . 187

Elias Saab and Paulette Saab, On Peł czyński's properties (V) and (V*) . . 205

Elmar Schrohe, The symbols of an algebra of pseudodifferential operators

Aart van Harten and Els Vader-Burger, Approximate Green functions as a tool to prove correctness of a formal approximation in a model of competing and diffusing species

Stephen Watson, Using prediction principles to construct ordered

continua 